www.nature.com/pj

\title{
Multipurpose soft-material SAXS/WAXS/GISAXS beamline at SPring-8
}

\author{
Hiroyasu Masunaga ${ }^{1}$, Hiroki Ogawa ${ }^{1}$, Takumi Takano ${ }^{1}$, Sono Sasaki ${ }^{1}$, Shunji Goto ${ }^{1}$, Takashi Tanaka ${ }^{2}$, \\ Takamitsu Seike ${ }^{1}$, Sunao Takahashi ${ }^{1}$, Kunikazu Takeshita ${ }^{1}$, Nobuteru Nariyama ${ }^{1}$, Haruhiko Ohashi ${ }^{1}$, \\ Toru Ohata $^{1}$, Yukito Furukawa ${ }^{1}$, Tomohiro Matsushita ${ }^{1}$, Yasuhide Ishizawa ${ }^{1}$, Naoto Yagi ${ }^{1}$, Masaki Takata ${ }^{1,2,3}$, \\ Hideo Kitamura ${ }^{2}$, Kazuo Sakurai ${ }^{4}$, Kohji Tashiro ${ }^{4}$, Atsushi Takahara ${ }^{4}$, Yoshiyuki Amamiya ${ }^{4}$, Kazuyuki Horie ${ }^{4}$, \\ Mikihito Takenaka ${ }^{4}$, Toshiji Kanaya ${ }^{4}$, Hiroshi Jinnai ${ }^{4}$, Hiroshi Okuda ${ }^{4}$, Isamu Akiba ${ }^{4}$, Isao Takahashi ${ }^{4}$, \\ Katsuhiro Yamamoto ${ }^{4}$, Masamichi Hikosaka ${ }^{4}$, Shinichi Sakurai ${ }^{4}$, Yuya Shinohara ${ }^{4}$, Akihiko Okada ${ }^{5}$ and \\ Yasunori Sugihara ${ }^{5}$
}

Scientific and engineering research on soft materials requires precise structural analysis to understand their hierarchical and fluctuating nature. A new beamline, the BL03XU frontier soft-material beamline, that is dedicated to scattering experiments on soft materials was recently installed at the third-generation synchrotron facility, SPring-8, in Japan. The BL03XU uses an in-vacuum undulator, and the photon flux of the obtained X-ray can reach $10^{13}$ photons $\mathrm{sec}^{-1}$, with an energy resolution of $\Delta E / E \approx 2 \times 10^{-4}$ at $12.4 \mathrm{keV}$. The BL03XU has two experimental hutches: a front one that is used for grazing-incidence scattering experiments and a second one for transmitting scattering experiments, which enables simultaneous small- and wide-angle X-ray scattering. The present paper introduces details about the instrument and some of the first scattering data measured at BL03XU, which reveals its cutting-edge design and high level of performance.

Polymer Journal (2011) 43, 471-477; doi:10.1038/pj.2011.18; published online 30 March 2011

Keywords: FSBL; scattering; SPring-8; synchrotron

\section{INTRODUCTION}

Soft materials represent a wide range of organic compounds that include polymers, colloids, surfactants and membranes. They exist in a variety of physical states that include semicrystallite, amorphous and glassy states, as well as liquid, thin film and micelle states. They are indispensably important materials in our daily lives as well as in modern industry. From a morphological point of view, they all share common features: fuzziness and hierarchy, which originate in the assembled structure of their constituent molecules and the timedependent fluctuations of their structure. Understanding the physical properties of soft materials and thus controlling and providing functionality requires accurate characterization of their fuzziness and hierarchy. Their constituent molecules are organized into mesoscopic structures that are larger than atoms and molecules, but much smaller than the macroscopic scale of the materials. The required observation scale can therefore range from $\sim 10^{-1}$ to $10^{4} \mathrm{~nm}$. The X-ray scattering technique, which has a combination of wide- and small-angle measurements, is one of the best tools to use in characterizing such systems. In addition, the fragile and hierarchical objects generally emit obscure, weak and complicated signals when compared with crystalline materials. Synchrotron light sources provide a great advantage in investigating these objects because they can detect very weak signals and enable us to carry out time-dependent experiments.

The new BL03XU beamline was recently constructed at SPring- 8 . It is dedicated for use in simultaneous small- and wide-angle X-ray scattering (SAXS/WAXS) measurements, small- and wide-angle $\mathrm{X}$-ray scattering under grazing incidence (GISAXS and GIWAXS), $\mathrm{X}$-ray diffraction $(\mathrm{XD})$ and X-ray reflectivity from soft materials. The construction of the beamline was directed by an industrial and academic joint organization called the frontier soft-material beamline (FSBL) consortium and with the profoundly kind technical cooperation of the Japan Synchrotron Radiation Research Institute (JASRI) and RIKEN Harima Institute. FSBL is an abbreviation of 'frontier soft-material beamline, a literal translation from a Japanese phrase meaning 'advanced soft-material beamline.' The consortium has rather distinctive features: there is no direct funding from the government, it consists of 19 Japanese corporations and academic members, the companies shared all of the expenses involved in the construction

\footnotetext{
${ }^{1}$ Japan Synchrotron Radiation Research Institute (JASRI/SPring-8), Sayo, Japan; ${ }^{2}$ RIKEN SPring-8 Center, Sayo, Japan; ${ }^{3}$ The University of Tokyo, Tokyo, Japan; ${ }^{4}$ FSBL (Representatives of the academic members), Sayo, Japan and ${ }^{5} \mathrm{FSBL}$ (Representatives of the industrial members), Sayo, Japan

Correspondence: Professor K Sakurai, Department of Chemistry and Biochemistry, The University of Kitakyushu, 1-1, Hibikino, Wakamatu-ku, Kitakyushu, Fukuoka 808-0135, Japan.

E-mail: sakura01@hibikino.ne.jp

Received 15 July 2010; revised 8 January 2011; accepted 13 January 2011; published online 30 March 2011
} 
and operation of the FSBL, each academic member was encouraged to collaborate with one of the industrial members to form 19 research groups before joining the consortium, and all the research groups provide two delegates (one an industrial and the other an academic member) to serve on the FSBL steering committee. The chair of the committee is an academic member, and the president of the FSBL consortium is elected from the industrial members. To advise the consortium and provide long-range insight from a broader perspective, the advisory committee consists of emeritus professors in the field. The consortium was first organized in 14 February 2008. Construction of the beamline commenced the same year and was completed at the end of March 2010.

SPring- 8 is one of the largest third-generation synchrotron radiation facilities in the world. The third-generation synchrotron X-ray sources are characterized by the exceptionally high brilliance of the photon beam, generally $>5 \mathrm{GeV}$, which is accomplished with the use of insertion devices. In 2006, a top-up operation was carried out at SPring- 8 , and a stable X-ray beam was then provided for users. ${ }^{1}$ The FSBL consortium decided to construct a new beamline dedicated to soft materials by taking advantage of that brilliance and the stability of the X-ray source. The present paper introduces the performance of the new beamline, reveals the features of the system and describes possibilities for future soft-material research in Japan using the new BL03XU beamline.

\section{OPTICS}

\section{Insertion device: undulator}

Undulators consist of two sets of permanent magnet arrays that face each other to generate 'quasi-monochromatic' electromagnetic radiation. Kitamura et al. ${ }^{2}$ developed a new undulator known as an 'invacuum undulator' in which the magnet arrays are inserted in a vacuum. ${ }^{3}$ With this design, the gap between the magnet arrays can be adjusted to be very narrow because there are no obstacles between them, and the undulator can thus generate a much stronger magnetic field than conventional insertion devices. The in-vacuum undulators were first installed in the storage ring at SPring-8 in 1996, and the first radiation was achieved in April 1997 at the BL47XU beamline. The FSBL uses a standard SPring- 8 in-vacuum undulator (magnet material: $\mathrm{NdFeB}$, periodic length: $32 \mathrm{~mm}$, number of periods: 140 , total length: $4.5 \mathrm{~m}$, available energy (first harmonic): $5-18 \mathrm{keV}$ ).
The minimum and maximum gap sizes are 8.1 and $45 \mathrm{~mm}$, respectively. The maximum magnetic field is $8.7 \mathrm{kG}$ at the minimum gap size. The pressure of all the devices reached below $1 \times 10^{-8} \mathrm{~Pa}$ after the bakeout process of the undulator.

\section{Monochromator}

The FSBL is equipped with a standard SPring- 8 double-crystal monochromator that uses a pair of Si crystals and provides a beam in the energy range of $6-35 \mathrm{keV}$ and a band pass of $\Delta E / E \sim 10^{-4}$ through use of Si (111) diffraction. Because of the high-radiation power (ca $580 \mathrm{Wmm}^{-2}$ ), two copper blocks cooled with circulated liquid nitrogen are attached to the silicon crystal for thermal regulation. The monochromator is located $42.0 \mathrm{~m}$ from the light source, as presented in Figure 1.

\section{Focusing mirror}

Two sets of perpendicularly aligned elliptical mirrors known as Kirkpartrick-Baez mirrors are used to focus the X-ray beam and eliminate its higher harmonics. ${ }^{4}$ At $6-16 \mathrm{keV}$, the glancing angles of both mirrors are adjusted to $4 \mathrm{mrad}$, and an $\mathrm{Rh}$ coat is used to eliminate the higher harmonics. At $16-20 \mathrm{keV}$, a Pt coat is used at the same glancing angles. At $20-35 \mathrm{keV}$, the angles are $2 \mathrm{mrad}$ and a Pt coat is used. The mirrors are located $53.2 \mathrm{~m}$ from the light source. By independently adjusting the elliptical curvatures of the two mirrors, the X-ray focus point can be located anywhere along the optical axis in the first and second hutches. For example, in the case of the front-end slit being $0.5 \times 0.3 \mathrm{~mm}$ (horizontal $\times$ vertical: $\mathrm{H} \times \mathrm{V}$ ) and with $12.4 \mathrm{keV}$ of X-ray energy, the mirror can focus a beam $0.170 \times 0.08 \mathrm{~mm} \mathrm{H} \times \mathrm{V}$ in size at the full-width at half maximum of the peak in the standard detector position of $69.5 \mathrm{~m}$ in the second hutch. Figure 2 presents an example of the beam image and its intensity profiles.

\section{Experimental hutches}

The FSBL has two experimental hutches: the first one is 6-m long and is designed for GIWAXS and GISAXS experiments that use a twodimensional (2D) detector, such as a cooled charge coupled device (CCD; additional details are given elsewhere), and the second is $10-\mathrm{m}$ long and $4.5 \mathrm{~m}$ high and is designed for SAXS and WAXS measurements. The second hutch has an additional large door $(3 \mathrm{~m}$ wide and $4 \mathrm{~m}$ tall) that enables the installation of a large kinematic mount or

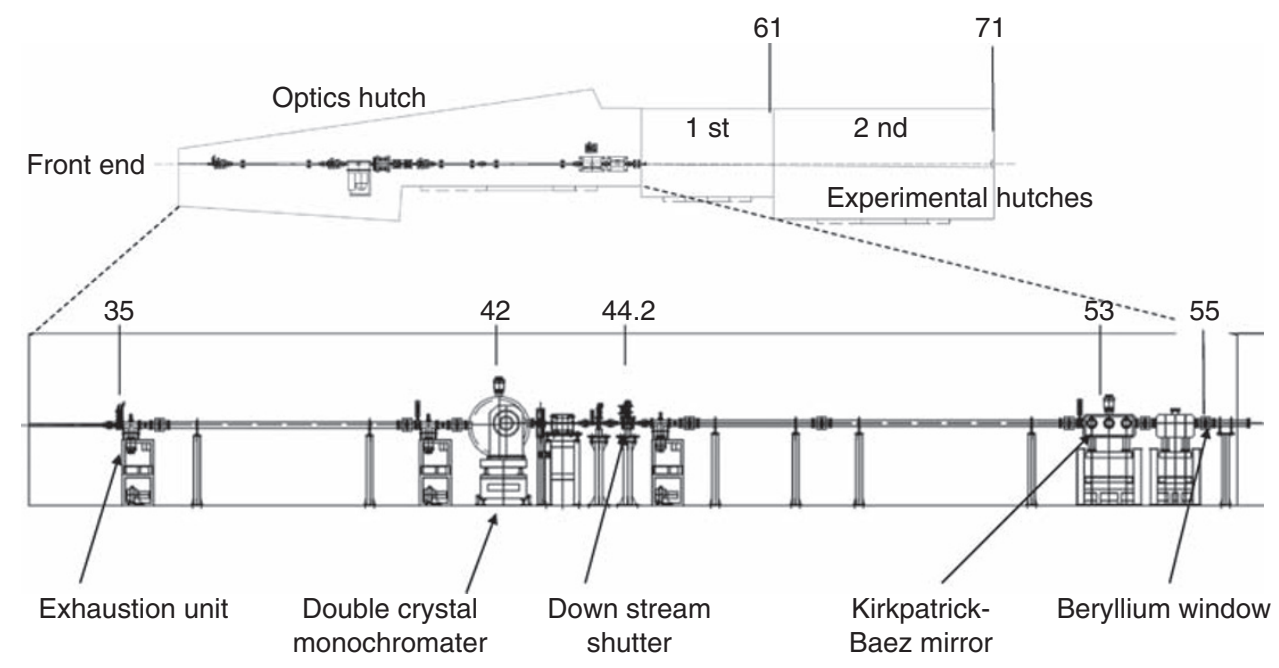

Figure 1 The optical layout of the BLO3XU beamline at SPring-8. An overhead view of the optical hutch. The first and second experimental hutches are at the top. A side view of the optical hutch is at the bottom. The numbers attached to the devices are the distance from the light source. 


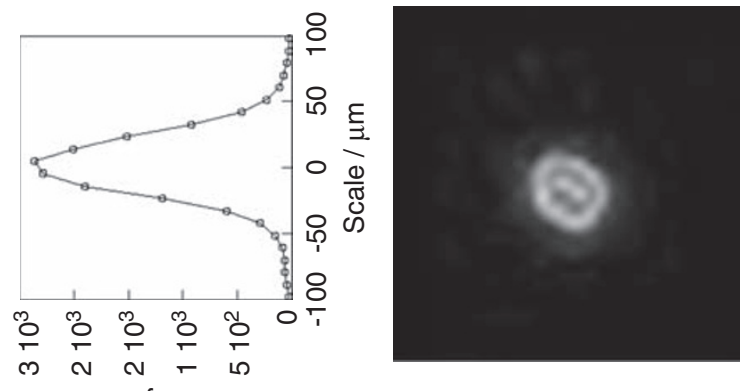

'n'e / Ki!suətul

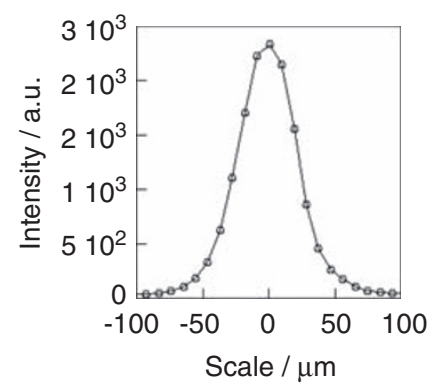

Figure $2 \mathrm{An}$ image of the $\mathrm{X}$-ray beam measured with a beam monitor AA40 $12 \mathrm{~m}$ from the $\mathrm{KB}$ mirror. The size of the first slit is $0.35 \times 0.35 \mathrm{~mm} \mathrm{H} \times \mathrm{V}$. The X-ray focus sizes are $\sim 50 \times 50 \mu \mathrm{m} \mathrm{H} \times \mathrm{V}$.

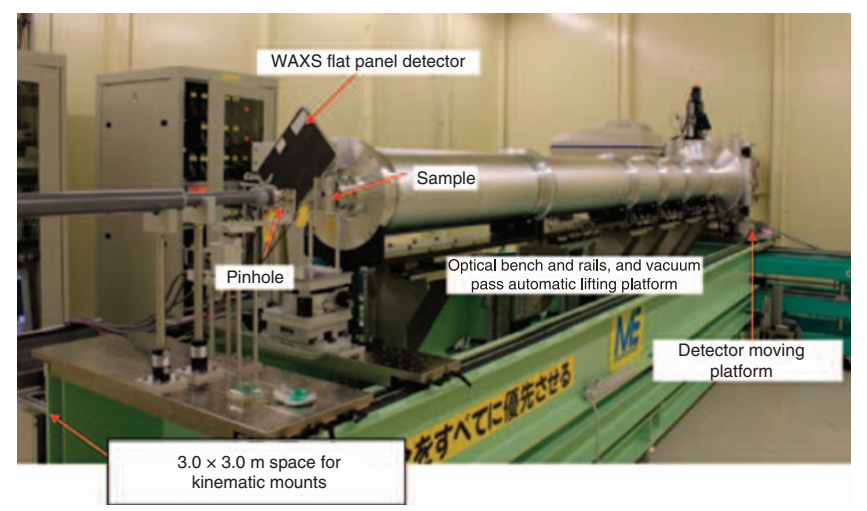

Figure 3 A typical setup for small-angle $X$-ray scattering (SAXS) and wideangle X-ray scattering (WAXS) measurements in the second hutch. A CCD camera used as the SAXS detector and an sample-to-detector distance of $4.1 \mathrm{~m}$.

processing instrument for in situ studies such as spinning and extruding. In the second hutch, a $3.0 \times 3.0 \mathrm{~m}$ space for kinematic mounts is positioned in front of the optical bench (Figure 3). A largecapacity exhaust air duct (ca $1 \mathrm{~m}$ area per sec) is joined to both hutches to enable organic solvents to be used inside them. The first hutch has an ion chamber (Ohyo Koken Kogyo, Tokyo, Japan, S-1329A) for measuring the intensity of X-rays, absorbers to attenuate the intensity, an X-ray shutter (Vincent Associates, San Francisco, CA, USA, UNIBLITZ XRS6) and a quadrant XY slit (Kohzu Precision, Kanagawa, Japan, GHM-13B) installed between the first and second hutches.

\section{$\mathrm{X}$-ray characteristics}

Use of the in-vacuum undulator and high-brilliance light source enables the photon flux to reach $10^{13}$ photons $\sec ^{-1}$, and an energy resolution of $\Delta E / E \sim 10^{-4}$ is achieved with a front-end slit of $0.5 \times 0.3 \mathrm{~mm} \mathrm{H} \times \mathrm{V}$ and a source energy of $E=12.4 \mathrm{keV}$. Tuning the

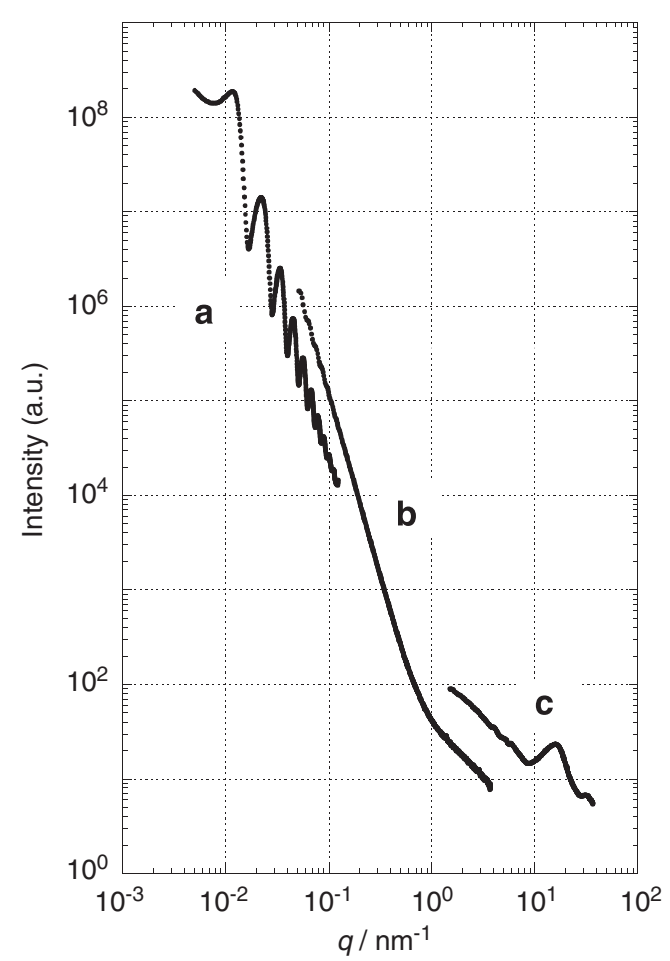

Figure 4 The scattering profile from $\mathrm{SiO}_{2}$ particles with a diameter of $570 \mathrm{~nm}$ measured with (A) sample-to-detector distance (SDD) $=9.3 \mathrm{~m}$ and $\lambda=0.2067 \mathrm{~nm}$, (B) $\mathrm{SDD}=2.2 \mathrm{~m}$ and $\lambda=0.1033 \mathrm{~nm}$, and (C) $\mathrm{SDD}=0.25 \mathrm{~m}$ and $\lambda=0.775 \mathrm{~nm}$. For greater convenience, the intensities were shifted vertically.

front-end slit and the two mirrors then enables the X-ray beam size to be altered to suit the different experiments. In most experiments, the first slits are used with an aperture of $0.35 \times 0.35 \mathrm{~mm} \mathrm{H} \times \mathrm{V}$. The X-ray focus size was observed to be $\sim 50 \times 50 \mu \mathrm{m} \mathrm{H} \times \mathrm{V}$ at the full-width at half maximum in optimized conditions, as presented in Figure 2. When the second hatch is used, parasitic scattering can be eliminated by use of a combination of the second and third slits and pinholes in front of the samples.

\section{Detectors and vacuum chamber in the second hutch}

The second hutch is equipped with a 2D X-ray image intensifier with a cooled CCD (Hamamatsu Photonics, Shizuoka, Japan, V7739P+ ORCA R2) and an imaging plate (IP) system (Rigaku, Tokyo, Japan, RAXIS VII) for measurements, as well as a beam monitor (Hamamatsu Photonics AA40). In most experiments, the detector position is fixed at $69.5 \mathrm{~m}$ from the light source. The three detectors are set on an $\mathrm{X}$-stage platform that can be moved horizontally in the direction perpendicular to the beam; thus, it takes several tens of seconds to exchange the detectors. Both the CCD and IP can be moved forward along the rails on the optical bench (4.5-m long) to adjust the sampleto-detector distance (SDD), as well as to move the guard pinholes, sample stages and the beam-stopper holder. This capability is of particular importance when kinematic mounts are used because the sample position cannot then be moved. The rails were constructed to be exactly parallel to the optical axis, and thus the position from the beam can be maintained within $100 \mu \mathrm{m}$ after being moved along the rails. This dimensional repeatability of the optical system can save enormous amounts of time when the SDD and other settings are changed. This has become increasingly important for the SAXS setup because the SDD is changed for almost every measurement. 
The choice of a suitable SDD should be determined by the characteristic length of the system to be studied. Except for a very short SDD, such as $10 \mathrm{~cm}$ for WAXS, the path behind the sample should be in an evacuated chamber to maintain the scattered X-ray intensity. One of the most important technical aspects of operating the new instrument is providing rapid and effortless reconfiguration of the vacuum chamber. All the vacuum tubes in the second hutch are stored in an optical bench and are retrieved mechanically. It generally takes several tens of minutes to change an SDD, including adjustment of the beam stopper. There are eight standard SDD setups that range from 6 to $0.25 \mathrm{~m}$. When a longer SDD is needed, the user can set a sample in the first hutch and locate the detector in the second hutch. In this case, the SDD can be elongated up to $10-\mathrm{m}$ long. The angle resolution (or $q$-resolution defined by $\Delta q / q$, where $q$ is the scattering vector) is determined by the divergent angle of the X-ray, the X-ray size at the detector surface, the scattering angle and the pixel resolution of the detector. With the FSBL and an SDD of $4.1 \mathrm{~m}$, a $\Delta q / q \approx 0.1$ for the small-angle region near the beam stopper is used; this can be decreased by increasing the scattering angle to $\Delta q / q \approx 6 \times 10^{-3}$ in the wide-angle region. With simultaneous SAXS and WAXS measurements, a flat-panel detector (Hamamatsu Photonics C9827DK-10) ${ }^{5}$ is used to make WAXS measurements. The flat panel is set several centimeters downstream of the sample ${ }^{6}$ so as not to obstruct the SAXS measurements. This then results (Figure 3 ) in an SAXS measurement of the $q$-range of $0.04-0.8 \mathrm{~nm}^{-1}$ and a WAXS measurement of the $q$-range of $5-30 \mathrm{~nm}^{-1}$.

Figure 4 provides the scattering profiles of silica particles with a diameter of $570 \mathrm{~nm}$ (Nippon Shokubai, Osaka, Japan, KE-P50) dispersed in epoxy resin and measured with a combination of three different SDDs and wavelengths (A: SDD $=9.3 \mathrm{~m}$ and $\lambda=0.2067 \mathrm{~nm}, \mathrm{~B}$ : $\mathrm{SDD}=2.2 \mathrm{~m}$ and $\lambda=0.1033 \mathrm{~nm}, \mathrm{C}: \mathrm{SDD}=0.25 \mathrm{~m}$ and $\lambda=0.775 \mathrm{~nm}$ ). The $q$-range covers $5 \times 10^{-3}$ to $30 \mathrm{~nm}^{-1}$. Because of the difference in angular resolution, profile A showed a clear amplitude of approximately $q=10^{-1} \mathrm{~nm}^{-1}$, whereas B shows a rather smeared one in the same $q$-range. It can be seen that the intensity, $I(q)$, satisfies the Porod a

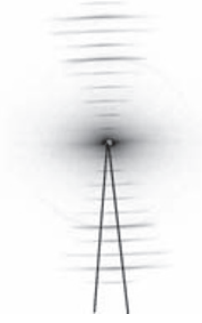

b

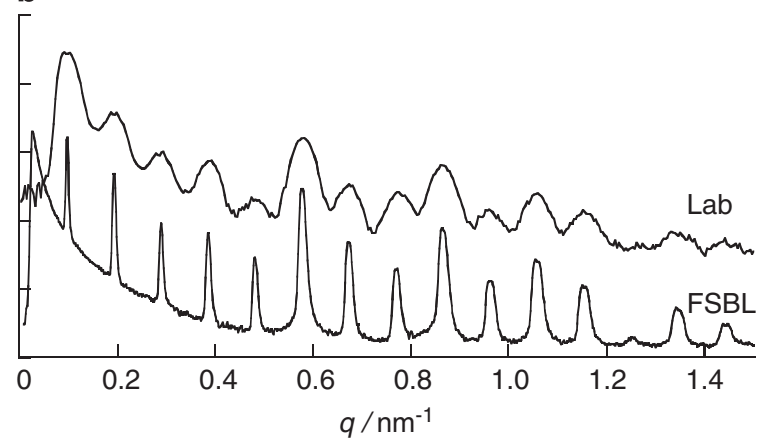

Figure 5 A two-dimensional small-angle X-ray scattering (SAXS) pattern from a collagen sample (a) and its profile (b) compared with one obtained with a conventional laboratory SAXS instrument.

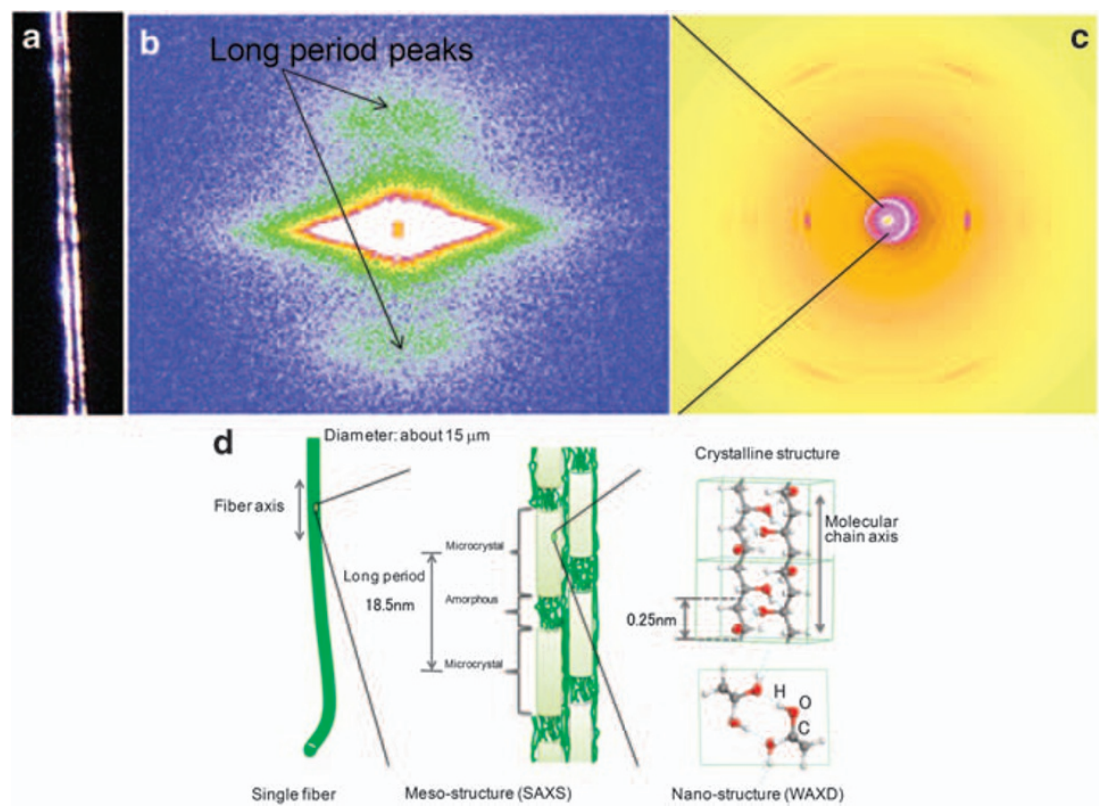

Figure 6 Small-angle X-ray scattering (SAXS) (b) and WAXS (c) patterns of a single Vinylon monofilament (a) $15 \mu \mathrm{m}$ in diameter produced in the late 1950 s to early 1960s. The long period of the crystallites was determined from the arrayed peaks in the SAXS pattern. The precise crystal structure was determined from the diffraction patterns in WAXS, as illustrated in $\mathbf{d}$. 
relationship of $I(q) \propto q^{-4}$ at $0.05<q<0.9 \mathrm{~nm}^{-1}$. The broad peak at $q=15 \mathrm{~nm}^{-1}$ is a reflection from the atomic structure of the $\mathrm{SiO}_{2}$.

\section{INSTRUMENTAL CAPABILITIES AND SCATTERING WITH STANDARD SAMPLES \\ Collagen}

Figure 5a shows a 2D SAXS pattern from dry chicken tendon collagen with $\lambda=0 . \mathrm{SDD}=4.25 \mathrm{~m}$ and $100 \mathrm{~nm}$ and an exposure time of $10 \mathrm{~s}$ with use of IP. An intensity profile was created by azimuthally averaging the indicated sector. The results are plotted in Figure 5b. With this setup, the smallest experimentally achieved $q$ for this SDD was $9.0 \times 10^{-3} \mathrm{~nm}^{-1}$. The first reflection of the collagen $(d=653 \AA)$ is clearly separated from the beam stopper, thus indicating excellent angular resolution. The present results are compared in Figure 5b with a profile from the same sample obtained with a conventional laboratory SAXS instrument, where $\lambda=0.154 \mathrm{~nm}(\mathrm{CuK} \alpha)$, generated with
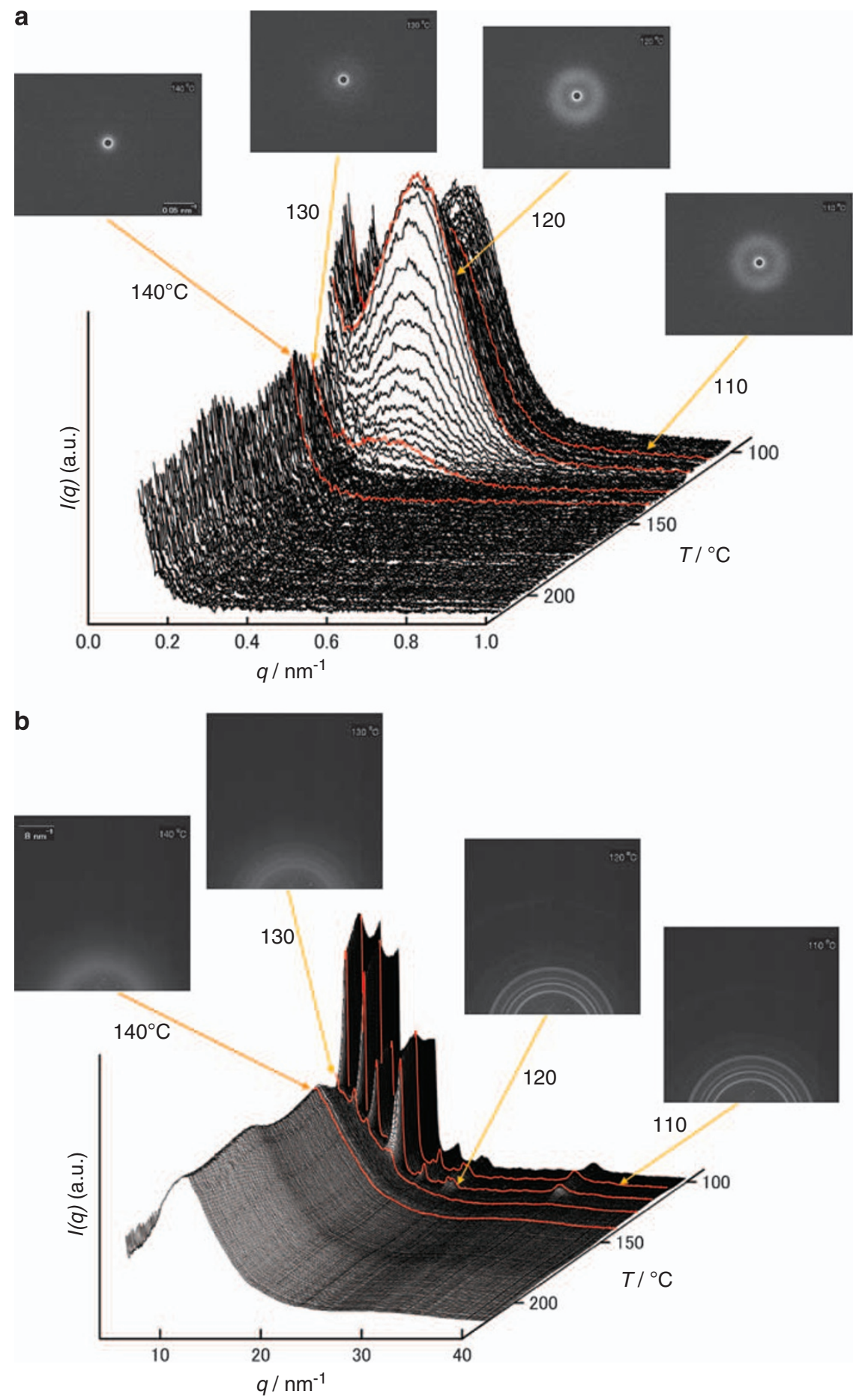

Figure 7 The temperature dependence of small-angle X-ray scattering (a) and WAXS (b) profiles of polypropylene upon cooling at a rate of $5^{\circ} \mathrm{C}$ min ${ }^{-1}$. 
a rotating anode $\mathrm{X}$-ray tube $(50 \mathrm{kV}$ and $100 \mathrm{~mA}), \mathrm{SDD}=1.14 \mathrm{~m}$, the exposure time was $600 \mathrm{~s}$, and the intensities were measured with a 2D position-sensitive detector (PSPC Hi-STAR). As expected, the FSBL profile has much narrower peaks and higher orders of diffraction than the conventional instrument, which demonstrates the high $q$-resolution of the FSBL. For example, the widths of the 6th peak are 0.018 and $0.09 \mathrm{~nm}^{-1}$ with the FSBL and the conventional instrument, respectively.

\section{Vinylon fiber}

Figure 6 shows the SAXS (B) and WAXD (C) patterns measured from a 'Vinylon' prototype monofilament (A). 'Vinylon' is the trade name of poly(vinyl alcohol) (PVA), and the present sample was made in 1955 at one of the first production plants in Japan. The SAXS pattern was measured using a $C C D$ camera with $S D D=3 \mathrm{~m}$, and a WAXD pattern with IP and an SDD of $0.3 \mathrm{~m}$. Combining the SAXS and WAXS data allows the hierarchical fiber structure to be determined, as presented in panel $\mathrm{D}$. The long-period peak was detected quite clearly with SAXS, revealing the existence of stacked lamellae at approximately the period of $18.5 \mathrm{~nm}$, and thus indicating that the fiber consists of a perpendicularly aligned crystalline and amorphous domain at the mesoscopic level. The WAXD data provided detailed information on the packing state of the planar-zigzag PVA chains in the crystalline lamellae. Analysis then revealed that the Vinylon structure at both the micro- and mesoscopic levels had been well controlled in the spinning and drowning processes so as to adapt it to particular uses or needs. It is quite amazing that the scientists and engineers involved in the industrialization of Vinylon somehow managed to discover how to tune its micro- and mesomorphology in the production process without even having directly observed them. The FSBL took just $20 \mathrm{~s}$ to reveal the orderly microstructure of the first proprietary Vinylon to have been manufactured half a century ago and demonstrated the high level of technology used to produce polymer fibers in those days.

PVA fiber was invented in Japan in 1939 by Prof Ichiro Sakurada, ${ }^{7}$ who is also well known as having discovered Mark-Houwink-Sakurada's equation, which describes the intrinsic viscosity of polymer solutions. The industrialization of PVA was first conducted independently by Kurashiki Rayon (now Kuraray) and Kanegabuchi Boseki (later Kanebo and then Kracie). The total amount of Vinylon produced worldwide has now reached over one million metric tons. The major markets for PVA include textile sizing agents, architectural and paper coatings, fibers and adhesives. Resin and films are produced from it, and it is primarily used in Asian countries, including Japan and China. PVA is a memorial material to the Japanese polymer community, and we selected it as a historical Vinylon fiber and used it as the first scattering sample in the FSBL. The sample was kindly supplied by the Institute for Chemical Research of Kyoto University via a recent author (T Kanaya), who is one of the descendants (member of the Takatsuki-kai group) of Prof Ichiro Sakurada.

\section{In situ measurement of polymer crystallization}

The crystallization process involved with isotactic polypropylene (Japan Polychem Corporation, weight-average molecular weight= $\left.2.9 \times 10^{5}\right)$. was investigated by performing simultaneous SAXS and WAXD measurements using the FSBL with an SDD of 1.5 and $0.0758 \mathrm{~m}$ and a CCD for the SAXS and an flat-panel detector for the WAXD. The isotactic polypropylene sample (thickness $=0.7 \mathrm{~mm}$ ) was heated to $215^{\circ} \mathrm{C}$ to melt it in advance, and it was then cooled at a rate of $5^{\circ} \mathrm{Cmin}^{-1}$ using a Linkam CSS450X. The SAXS and WAXD data were measured during the continuous cooling process (non- isothermal crystallization). The exposure times were 100 and $1000 \mathrm{~ms}$ with the SAXS and WAXD, respectively. The interval time was $6 \mathrm{~s}$. Typical 2D SAXS and WAXS patterns are presented in Figure 7. In spite of the rather short exposure times, quite clear scattering images were successfully observed. Sector-averaging the data then provided the temperature dependence of the profiles, as presented in the figure. As has been reported previously, ${ }^{8}$ the SAXS showed a broad peak of approximately $q=0.2 \mathrm{~nm}^{-1}$ because of the density fluctuation in the molten state at $130{ }^{\circ} \mathrm{C}$. This broad peak then developed into a long-period peak after crystallization and shifted to a higher $q$, at which point the WAXS pattern then began to display crystalline peaks. As has already been reported, domain structures were created in the cooling process after melting, and this resulted in the development of crystalline domains with regular helices. For a more detailed discussion, it would be more useful to perform simultaneous SAXS and WAXD measurements and use vibrational spectroscopic data.

\section{PERSPECTIVES OF FSBL}

The FSBL consortium held a ceremony on 4 February 2010, to celebrate the completion of the beamline. Beginning in April, we also made time available for use of the FSBL. In addition, a new type of business-academia collaboration was launched for each group. This powerful structural analysis tool can be expected to tighten the relationship between scattering and soft-material research and to expand our scientific and technical horizons in this field. The FSBL enables more elaborate structural analysis and thus more explicit visualization of structures at the molecular level. The strong X-ray power should enable us to observe dynamic structural changes that take place in soft materials at the micro- and mesoscopic levels and could reveal new principles and rules to use in controlling them. These new insights could also then suggest new directions and ideas to be used in the molecular design of soft materials.

\section{CONCLUSIONS}

Construction of the FSBL was carried out as a joint project between industrial and academic members of an FSBL consortium, with the technical support of JASRI and the RIKEN Harima Institute. It was completed at the end of March in 2010. Because of the in-vacuum undulator and the highly brilliant light source, the photon flux of the obtained X-ray can reach $10^{13}$ photons $\mathrm{sec}^{-1}$, and an energy resolution of $\Delta E / E \approx 2 \times 10^{-4}$ has now been achieved. There are two experimental hutches: the front one is for GISAXS and GIWAXS measurements, and the second is for simultaneous SAXS and WAXS measurements. Preliminary measurements on collagen, Vinylon and polypropylene revealed the high performance of the BL03XU FSBL beamline.

\section{ACKNOWLEDGEMENTS}

We thank the 19 corporations comprising the FSBL consortium that funded the FSBL construction: Asahi Kasei Corporation; Kwansei Gakuin University; Canon; Kuraray; Showa Denko KK; Sumitomo Chemical; Sumitomo Rubber Industries; Sumitomo Bakelite; Denso Corporation; Toyobo; Toray Industries; Nitto Denko Corporation; Bridgestone Corporation; Mitsui Chemicals; Mitsubishi Chemical Corporation; Mitsubishi Rayon; Yokohama Rubber; Teijin Limited; and DIC Corporation. We also thanks to the many academic and industrial members who participated in the construction of the FSBL.

\footnotetext{
1 Tanaka, H., Adachi, M., Aoki, T., Asaka, T., Baron, A., Daté, S., Fukami, K., Furukawa, Y., Hanaki, H., Hosoda, N., Ishikawa, T., Kimura, H., Kobayashi, K., Kobayashi, T., Kohara, S., Kumagai, N., Masaki, M., Masuda, T., Matsui, S., Mizuno, A., Nakamura, T., Nakatani, T., Noda, T., Ohata, T., Ohkuma, H., Ohshima, T., Oishi, M., Sasaki, S., Schimizu, J., Shoji, M., Soutome, K., Suzuki, M., Suzuki, S., Suzuki, Y., Takano, S.,
} 
Takao, M., Takashima, T., Takebe, H., Takeuchi, A., Tamura, K., Tanaka, R., Tanaka, Y., Taniuchi, T., Taniuchi, Y., Tsumaki, K., Yamashita, A., Yanagida, K., Yoda, Y., Yonehara, H., Yorita, T., Yoshioka, M. \& Takata, M. Stable top-up operation at SPring-8. J. Synchrotron Radiat. 13, 378-391 (2006).

2 Yamazaki, H., Yabashi, M., Tamasaku, K., Yoneda, Y., Goto, S., Mochizuki, T. \& Ishikawa, T. SPring-8 standard X-ray monochromator: alignment of rotated-inclined geometry. Nucl. Instrum. Methods Phys. Res. A 467-468, 643-646 (2001).

3 Tanaka, T. J. Jpn. Soc. Synchrotron Rad. Res. 3, 251 (1997).

4 Mimura, H., Matsuyama, S., Yumoto, H., Hara, H., Yamamura, K., Sano, Y., Shibahara, M., Endo, K., Mori, Y., Nishino, Y., Tamasaku, K., Yabashi, M., Ishikawa, T. \& Yamauchi, K. Hard X-ray diffraction-limited nanofocusing with kirkpatrick-baez mirrors. Jpn. J. Applied Phys. 44, L539.
5 Yagi, N. \& Inoue, K. CMOS flatpanel detectors for SAXS/WAXS experiments. J. Appl. Crystallogr. 40, s439-s441 (2007).

6 Masunaga, H., Sasaki, S., Tashiro, K., Hanesaka, M., Takata, M., Inoue, K., Ohta, N. \& Yagi, N. Development of synchrotron DSC/WAXD/SAXS simultaneous measurement system for polymeric materials at the BL4OB2 in SPring-8 and its application to the study of crystal phase transitions of fluorine polymers. Polym. J. 39, 1281-1289 (2007).

7 Sakurada, I. Polyvinyl Alcohol Fibers (Marcel Dekker, Inc., New York, 1985).

8 Reddy, K. R., Tashiro, K., Sakurai, T., Yamaguchi, N., Sasaki, S., Masunaga, H. \& Takata, M. Isothermal crystallization behavior of isotactic polypropylene H/D blends as viewed from time-resolved FTIR and synchrotron SAXS/WAXD measurements. Macromolecules 42, 4191-4199 (2009). 